\title{
Impulsive synchronization of Lipschitz chaotic systems
}

\author{
Yen-Sheng Chen ${ }^{a}$, Chien-Cheng Chang ${ }^{a, b, *}$ \\ a Division of Mechanics, Research Center for Applied Sciences, Academia Sinica, Taipei 115, Taiwan, ROC \\ ${ }^{\mathrm{b}}$ Institute of Applied Mechanics \& Taida Institute of Mathematical Sciences, National Taiwan University, Taipei 106, Taiwan, ROC
}

Accepted 31 August 2007

\begin{abstract}
Impulsive method is suitable for digital implementation of secure communication based on chaos synchronization. In the present study, it is assumed that the system satisfies the local Lipschitz condition where a Lipschitz constant is estimated a priori. An impulsive controller is shown to achieve synchronization of chaotic systems in the sense of exponential stability under one restriction relation (criterion). The Duffing two-well and the Rössler systems were simulated to illustrate the theoretical analysis.

(C) 2007 Elsevier Ltd. All rights reserved.
\end{abstract}

\section{Introduction}

Chaotic systems were thought difficult to be synchronized or controlled in the past as chaotic systems exhibit sensitive dependence on initial conditions. Since 1980s, researchers have realized that chaotic motions can be synchronized through a feedback mechanism [1] or by linking two systems with common signals [2], as well as controlling chaos is also possible [3,4]. In the past decade, we have seen a rapid growth of theoretical and experimental studies on chaos synchronization. This is partly because chaos synchronization has potential applications in secure communication [5], information processing [6], pattern formation [7], etc.

Synchronization means that the state of the response system eventually approaches that of the driving system. Two kinds of chaos synchronization are most often discussed: (1) The master-slave scheme, introduced by Pecora and Carroll [2], consists of two identical systems with different initial conditions. The master system is chaotic while some state variables of the slave system are replaced by the corresponding ones of the master system. Synchronization occurs if and only if all the (conditional) Lyapunov exponents, quantifying stretching properties of some trajectories [8], to the unreplaced state variables are negative [2,9]. (2) The second kind of synchronization, the coupling scheme, deals with two identical chaotic systems except coupling terms which can be either unidirectional or bidirectional. Under certain conditions the response system may eventually evolve into the same orbit of the driving system.

Synchronization discussed above is called complete synchronization or simply synchronization. There are other types of synchronization such as generalized synchronization, phase synchronization, lag, and anticipated synchronization.

\footnotetext{
${ }^{*}$ Corresponding author.

E-mail addresses: ysc@gate.sinica.edu.tw (Y.-S. Chen), mechang@gate.sinica.edu.tw (C.-C. Chang).
} 
Generalized synchronization means that evolving orbits of the response system approach the image of those to the driving system under a function relationship $[10,11]$. This function is not necessarily defined on the whole phase space but on its attractor only. Phase synchronization is that the phases of two systems come closely $[12,13]$ as time evolves though the amplitudes remain almost uncorrelated. Lag (anticipated) synchronization means that the state of the response system approaches eventually that of the driving system with a time delay [13] (lead [14]). Furthermore, synchronization of time-delayed systems and related issues can also be found in the literatures $[15,16]$.

In this work, we propose an impulsive controller to achieve synchronization of chaotic systems. Impulsive control method fits digital implementation of secure communication based on chaos synchronization. It is therefore important to understand how impulsive control accomplishes chaos synchronization. This method is not only applied to chaos synchronization but also to medicine, biology, and economics. Some other impulsive designs, such as comparison method $[17,18]$ and linear feedback [19] may also achieve synchronization of chaotic systems.

The systems considered in the present study are assumed to satisfy the local Lipschitz condition where a Lipschitz constant is estimated a priori. Next, impulse intervals and a feedback gain matrix are elected according to one single restriction relation (criterion) which connects the Lipschitz constant, the rate of convergence, the gain matrix, and impulse intervals. The estimated error state vector is proven to converge in the sense of exponential stability. The criterion is easy to implement in locating the admissible region of control parameters to ensure the occurrence of synchronization. Finally, the Duffing two-well and the Rössler systems are simulated to illustrate the validity of the theoretical analysis.

\section{Theoretical analysis}

Consider a system

$$
\dot{\mathbf{x}}=\mathbf{f}(t, \mathbf{x}),
$$

and an impulsive controlled system

$$
\begin{aligned}
& \dot{\hat{\mathbf{x}}}=\mathbf{f}(t, \hat{\mathbf{x}}), t \neq t_{i}^{+}, \\
& \left.\Delta \hat{\mathbf{x}}\right|_{t=t_{i}}=\hat{\mathbf{x}}\left(t_{i}^{+}\right)-\hat{\mathbf{x}}\left(t_{i}^{-}\right)=: U(i), t=t_{i}^{+},
\end{aligned}
$$

where $\mathbf{x}, \hat{\mathbf{x}} \in \mathbb{R}^{N}$ denote the state vectors, $\hat{\mathbf{x}}\left(t_{0}^{+}\right)=\hat{\mathbf{x}}_{0}$ and $\Omega$ is a domain containing the origin. The function $\mathbf{f}: \Omega \subset \mathbb{R} \times \mathbb{R}^{N} \rightarrow \mathbb{R}^{N}$ satisfies the Lipschitz condition $\left\|\mathbf{f}\left(t, \mathbf{x}_{1}\right)-\mathbf{f}\left(t, \mathbf{x}_{2}\right) \mid \leqslant L\right\| \mathbf{x}_{1}-\mathbf{x}_{2} \|$ for all $\left(t, \mathbf{x}_{1}\right)$ and $\left(t, \mathbf{x}_{2}\right)$ in $\Omega$ with a Lipschitz constant $L$. The time sequence $\left\{t_{i}\right\}$ of impulse instants is $0<t_{1}<t_{2}<\cdots<t_{i}<\cdots$, and $t_{i} \rightarrow \infty$ as $i \rightarrow \infty$. The present work requires

$$
\left|t_{i}-t_{i-1}\right| \leqslant T<\infty, \quad i=1,2, \ldots
$$

Let the state error be $\mathbf{e}(t)=\hat{\mathbf{x}}(t)-\mathbf{x}(t)$, then

$$
\Delta \mathbf{e}\left(t_{i}\right):=\left[\hat{\mathbf{x}}\left(t_{i}^{+}\right)-\mathbf{x}\left(t_{i}^{+}\right)\right]-\left[\hat{\mathbf{x}}\left(t_{i}^{-}\right)-\mathbf{x}\left(t_{i}^{-}\right)\right]=\left.\Delta \hat{\mathbf{x}}\right|_{t=t_{i}},
$$

for $i=1,2, \ldots$. Notice that system (1) is continuous, $\mathbf{x}\left(t_{i}^{+}\right)-\mathbf{x}\left(t_{i}^{-}\right)$is a zero vector at $t=t_{i}$ for $i=1,2, \ldots$ The error dynamical system becomes

$$
\begin{aligned}
& \dot{\mathbf{e}}=\mathbf{f}(t, \mathbf{x}+\mathbf{e})-\mathbf{f}(t, \mathbf{x}), t \neq t_{i}^{+}, \\
& \Delta \mathbf{e}\left(t_{i}\right)=\left.\Delta \hat{\mathbf{x}}\right|_{t=t_{i}}, t=t_{i}^{+}, \quad i=1,2, \ldots
\end{aligned}
$$

As a result, $\Delta \mathbf{e}\left(t_{i}\right)=U(i)$ at $t=t_{i}^{+}$for $i=1,2, \ldots$ The purpose is to choose an appropriate impulsive controller $U(i)$ such that the error state $\mathbf{e}(t)=0$ is asymptotically stable, i.e. system (2) synchronizes to system (1).

In the present study, we choose the impulsive controller in the form of negative feedback as

$$
U(i)=\Gamma(i) \mathbf{e}\left(t_{i}^{-}\right), \quad i=1,2, \ldots,
$$

where the gain matrix $\Gamma(i)$ actuated at $t=t_{i}^{+}$is to be determined. A criterion of chaos synchronization for systems (1) and (2) is provided by the following theorem.

Theorem. The error state $\mathbf{e}=0$ is exponentially stable if the impulsive controller is $U(i)=\Gamma(i) \mathbf{e}\left(t_{i}^{-}\right)$, where $\Gamma(i)$ satisfies

$$
\|I+\Gamma(i)\| \leqslant \mathrm{e}^{-\alpha / 2-L\left(t_{i}-t_{i-1}\right)},
$$

with $\alpha>0$. 
Proof. Choose a function

$$
V=\frac{1}{2} \mathbf{e}^{\mathrm{T}} \mathbf{e}=\frac{1}{2}\|\mathbf{e}\|^{2} .
$$

Differentiating $V$ with respect to $t$ yields an estimate

$$
\dot{V}=\mathbf{e}^{\mathrm{T}} \dot{\mathbf{e}}=\mathbf{e}^{\mathrm{T}}[\mathbf{f}(t, \mathbf{x}+\mathbf{e})-\mathbf{f}(t, \mathbf{x})] \leqslant L\|\mathbf{e}\|^{2}=2 L V,
$$

where we have used the Cauchy-Schwarz inequality and the Lipschitz condition for $\mathbf{f}$. Consider time intervals in which the system evolves excluding impulse instants, we obtain

$$
V(t) \leqslant V\left(t_{i-1}^{+}\right) \mathrm{e}^{2 L\left(t-t_{i-1}\right)},
$$

for $t \in\left(t_{i-1}, t_{i}\right], i=1,2, \ldots$. In light of (5), we have

$$
\begin{aligned}
V\left(t_{i}^{+}\right) & =\frac{1}{2}\left[\mathbf{e}\left(t_{i}^{-}\right)+\Delta \mathbf{e}\left(t_{i}\right)\right]^{\mathrm{T}}\left[\mathbf{e}\left(t_{i}^{-}\right)+\Delta \mathbf{e}\left(t_{i}\right)\right] \\
& =\frac{1}{2}\left[(I+\Gamma(i)) \mathbf{e}\left(t_{i}^{-}\right)\right]^{\mathrm{T}}\left[(I+\Gamma(i)) \mathbf{e}\left(t_{i}^{-}\right)\right] \\
& =\frac{1}{2}\left\|(I+\Gamma(i)) \mathbf{e}\left(t_{i}^{-}\right)\right\|^{2} \\
& \leqslant \frac{1}{2}\|I+\Gamma(i)\|^{2}\left\|\mathbf{e}\left(t_{i}^{-}\right)\right\|^{2} \\
& =\|I+\Gamma(i)\|^{2} V\left(t_{i}^{-}\right), \quad i=1,2, \ldots
\end{aligned}
$$

At $t=t_{1}^{+}$, combining (7) and (8) yields

$$
V\left(t_{1}^{+}\right) \leqslant\|I+\Gamma(1)\|^{2} V\left(t_{1}^{-}\right) \leqslant V\left(t_{0}^{+}\right)\|I+\Gamma(1)\|^{2} \mathrm{e}^{2 L\left(t_{1}-t_{0}\right)} .
$$

Using (7) again, we have for $t \in\left(t_{1}, t_{2}\right]$

$$
V(t) \leqslant V\left(t_{1}^{+}\right) \mathrm{e}^{2 L\left(t-t_{1}\right)} \leqslant V\left(t_{0}^{+}\right)\|I+\Gamma(1)\|^{2} \mathrm{e}^{2 L\left(t-t_{1}\right)} \mathrm{e}^{2 L\left(t_{1}-t_{0}\right)} .
$$

Repeating the same process yields for $t \in\left(t_{i}, t_{i+1}\right]$

$$
V(t) \leqslant V\left(t_{0}^{+}\right) \mathrm{e}^{2 L\left(t-t_{i}\right)} \prod_{k=1}^{i}\|I+\Gamma(k)\|^{2} \mathrm{e}^{2 L\left(t_{k}-t_{k-1}\right)} .
$$

If $\Gamma(i)$ satisfies $\|I+\Gamma(i)\|^{2} \mathrm{e}^{2 L\left(t_{i}-t_{i-1}\right)} \leqslant \mathrm{e}^{-\alpha}$ with $\alpha>0$, then the estimate of $V$ becomes $V(t) \leqslant V\left(t_{0}^{+}\right) \mathrm{e}^{2 L\left(t-t_{i}\right)} \mathrm{e}^{-\alpha i}$.

Since $\mathrm{e}^{2 L\left(t-t_{i}\right)}$ is bounded due to the assumption (3), the function $V(t) \leqslant V\left(t_{0}^{+}\right) \mathrm{e}^{2 L\left(t-t_{i}\right)} \mathrm{e}^{-\alpha i} \rightarrow 0$ as $i \rightarrow \infty(t \rightarrow \infty)$. Hence, $\|\mathbf{e}(t)\|=\sqrt{2 V(t)}$ approaches zero exponentially.

From the proof, we see that the error dynamics converges at the rate not less than $\mathrm{e}^{-\alpha i}$, and therefore $\alpha$ is called the rate of synchronization. Although the rate of synchronization can be chosen differently for each impulsive interval, we adopt a uniform rate of synchronization for convenience. Furthermore, $\alpha$ is selected in accordance with actual requirement. To apply the theorem we have to estimate a Lipschitz constant $L$, choose the rate of synchronization $\alpha$, and then define the impulse interval $t_{i}-t_{i-1}$ and the feedback gain matrix $\Gamma(i)$ according to (6). If the gain matrix is diagonal as

$$
\Gamma(i)=\operatorname{diag}\left\{\gamma_{1}(i) \quad \cdots \quad \gamma_{j}(i) \quad \cdots \quad \gamma_{n}(i)\right\},
$$

then the condition (6) becomes

$$
\left[1+\gamma_{j}(i)\right]^{2} \leqslant \mathrm{e}^{-\alpha-2 L\left(t_{i}-t_{i-1}\right)}, \alpha>0,
$$

which is further recast into

$$
-\mathrm{e}^{-\alpha / 2-L\left(t_{i}-t_{i-1}\right)}-1 \leqslant \gamma_{j}(i) \leqslant \mathrm{e}^{-\alpha / 2-L\left(t_{i}-t_{i-1}\right)}-1,
$$

$\alpha>0$ for $j=1, \ldots, n$. In other words, synchronization is ensured if each diagonal element of the gain matrix satisfies the previous inequality. Since $\alpha, L$ and $t_{i}-t_{i-1}$ must be positive, we have $0<\mathrm{e}^{-\alpha / 2-L\left(t_{i}-t_{i-1}\right)}<1$. This leads to that the components of the diagonal gain matrix at each $t_{i}^{+}$meet $-2<\gamma_{j}(i)<0$ for $j=1, \ldots, n$.

A corollary of previous theorem follows immediately if all the impulse intervals are constant, i.e. $t_{i}-t_{i-1}=\tau$, and the gain matrix remains $\Gamma(i)=\Gamma$ at each impulse instant. 
Corollary. The error state $\mathbf{e}=0$ is exponentially stable if the impulsive controller $U(i)=\Gamma \mathbf{e}\left(t_{i}^{-}\right)$, where $\Gamma=\operatorname{diag}\left\{\begin{array}{lllll}\gamma_{1} & \cdots & \gamma_{j} & \cdots & \gamma_{n}\end{array}\right\}$ satisfies

$$
-e^{-\alpha / 2-L \tau}-1 \leqslant \gamma_{j} \leqslant e^{-\alpha / 2-L \tau}-1,
$$

with $\alpha>0$.

We can reformulate (9) to get the upper bound of the impulse interval as

$$
t_{i}-t_{i-1} \leqslant-\left[\alpha+\ln \left[1+\gamma_{j}(i)\right]^{2}\right] / 2 L .
$$

This gives a restriction relation between $t_{i}-t_{i-1}$ and $\gamma_{j}(i)$. In the simplest case $\gamma_{j}(i)=\gamma$, Fig. 1 depicts some level curves for $L=1,3,9,17$ and $L=24$ with $\alpha=0.2$ when the equality in (11) holds. The area below each level curve is the admissible region where we have feasible control gains and impulse intervals. The level curves are therefore called curves of restriction. From the proof of the theorem, a large $\alpha$ improves the rate of synchronization, but also shrinks the admissible region of $\gamma$ and $t_{i}-t_{i-1}$. For example, consider the case $L=1$ with $\alpha=0.2$. Fig. 1 shows the curve of restriction in the bold line as $\alpha$ becomes 1 . The alternative bold line pinpoints the limiting situation as $\alpha=0$ for $L=1$.

Remark. In the ideal case of $\Gamma(i)=-I$ at some instant $t_{i}^{+}$, the inequalities (6), (10), and (11) always hold and the impulse interval can be infinite. It means that the state of system (2) at $t=t_{i}^{+}$is reset to be identically equal to that of system (1). Consequently, evolutions of these two systems coincide after $t_{i}^{+}$. Fix the impulse interval $t_{i}-t_{i-1}$, a larger admissible area allows us to choose the control gain near its bounds -2 and 0 . This implies that we can achieve synchronization under smaller control effort.

If the dynamics of system (1) is in an equilibrium state, the problem reduces to the one of control stabilization for which the present method still works. In practical applications, the control parameters cannot be perfectly accomplished. It is therefore important to know margins of control parameters where the control objective is still

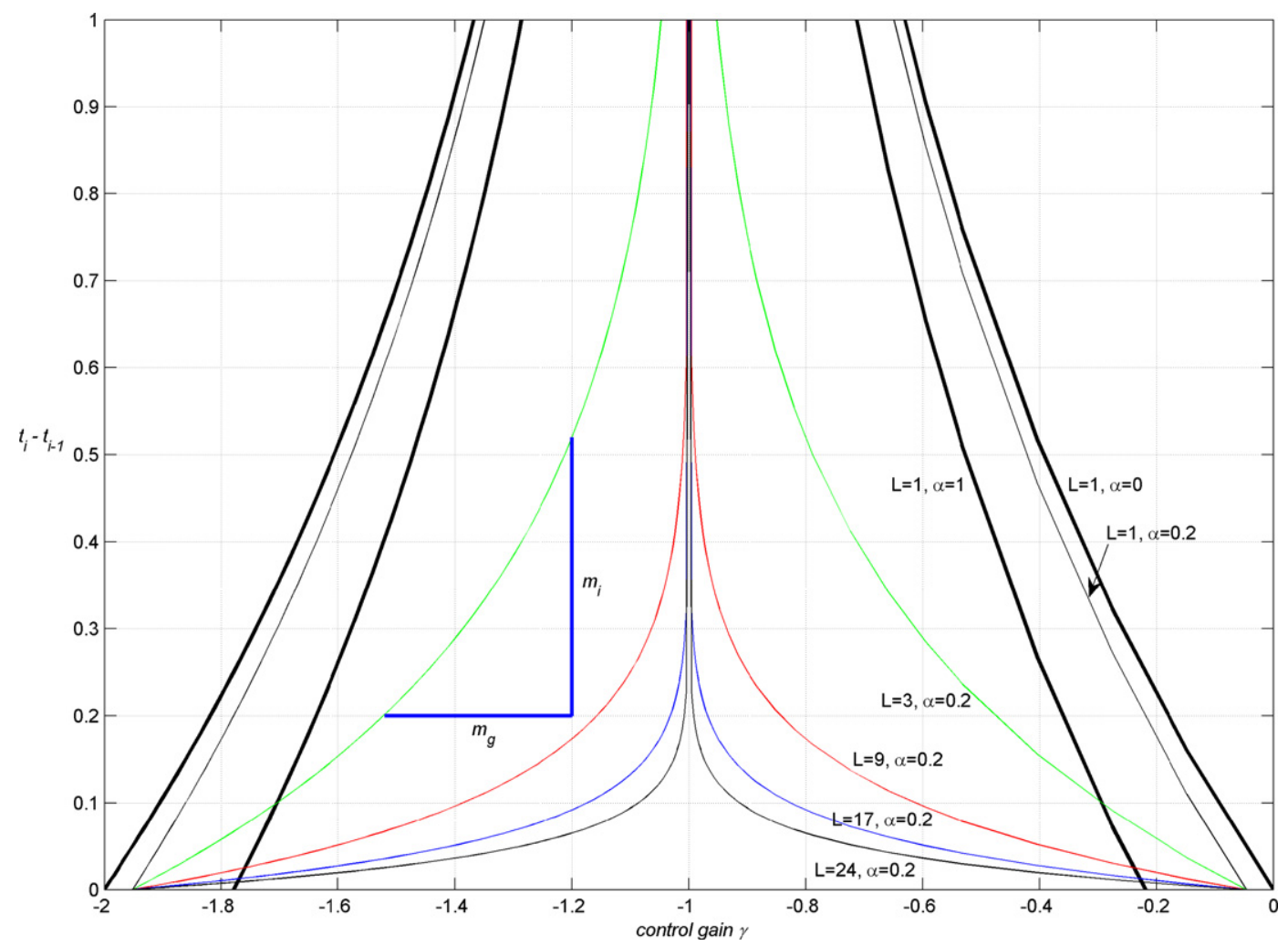

Fig. 1. Curves of restriction of $t_{i}-t_{i-1}$ and $\gamma$ for some Lipschitz constants $L=1,3,9,17$, and 24 with $\alpha=0.2$. Bold lines indicate curves of restriction for $\alpha=0$ and 1 as $L=1$. 
achieved. According to (9) or (10), once we select a pair of feasible control gain and impulse interval in the admissible region: the gain margin $m_{\mathrm{g}}$ is the horizontal line segment from this point to the restriction curve as the impulse interval is fixed, and the impulse interval margin $m_{i}$ is the vertical counterpart as the control gain is fixed. Here the margins warrant stabilization under some small disturbances of control parameters corresponding to their margins, but we do not mean that the error system must be unstable as the parameter perturbation exceeds its margin. A pair of $m_{\mathrm{g}}$ and $m_{i}$ is shown in Fig. 1.

\section{Numerical illustrations}

Although based on non-autonomous systems, the criteria developed in the previous section are also applicable to autonomous systems. The Duffing two-well and Rössler systems, non-autonomous and autonomous systems respectively, will be simulated to demonstrate the theoretical analysis.

(A) The Duffing two-well oscillator. The equations are given by

$$
\begin{aligned}
& \dot{x}=y=: f_{1}(\mathbf{x}), \\
& \dot{y}=-x^{3}+x-b y+A \sin \omega t=: f_{2}(\mathbf{x}), \\
& \dot{\hat{x}}=\hat{y} \\
& \dot{\hat{y}}=-\hat{x}^{3}+\hat{x}-b \hat{y}+A \sin \omega t, \\
& \left.\Delta \hat{x}\right|_{t=t_{i}}=\gamma_{1} e_{1}, \\
& \left.\Delta \hat{y}\right|_{t=t_{i}}=\gamma_{2} e_{2}, \quad i=1,2, \ldots,
\end{aligned}
$$

where the parameters $b=0.25, A=0.4$ and $\omega=1$ with the initial condition $\left[\begin{array}{llll}x_{0} & y_{0} & \hat{x}_{0} & \hat{y}_{0}\end{array}\right]^{\mathrm{T}}=\left[\begin{array}{llll}0.2 & 1 & 0 & 0\end{array}\right]^{\mathrm{T}}$ ensure the existence of chaotic attractor. Applying the Cauchy-Schwarz inequality to estimate a Lipschitz constant, we have for any $\mathbf{x}_{1}=\left[\begin{array}{ll}x_{1} & y_{1}\end{array}\right]^{\mathrm{T}}, \mathbf{x}_{2}=\left[\begin{array}{ll}x_{2} & y_{2}\end{array}\right]^{\mathrm{T}}$,

$$
\begin{aligned}
\left|f_{1}\left(\mathbf{x}_{2}\right)-f_{1}\left(\mathbf{x}_{1}\right)\right| \leqslant & \left\|\mathbf{x}_{2}-\mathbf{x}_{1}\right\|, \\
\left|f_{2}\left(\mathbf{x}_{2}\right)-f_{2}\left(\mathbf{x}_{1}\right)\right|= & \left|e_{1}-b e_{2}-x_{2}^{3}+x_{1}^{3}\right| \\
& \leqslant\left|e_{1}-b e_{2}-x_{2}^{2} e_{1}-x_{1}\left(x_{2}+x_{1}\right) e_{1}\right| \\
& \leqslant\left\|\left[1+3 B_{1}^{2} b\right]\right\|\left\|\mathbf{x}_{2}-\mathbf{x}_{1}\right\|,
\end{aligned}
$$

where $\left|x_{i}(t)\right| \leqslant B_{1}, \quad\left|y_{i}(t)\right| \leqslant B_{2}, \quad \forall t>t_{0}, \quad i=1,2$. Hence, one Lipschitz constant can be chosen to be $L=\sqrt{1+\left\|\left[1+3 B_{1}^{2} b\right]\right\|^{2}}$. From numerical simulation, we obtain $B_{1}=1$ and $B_{2}=1$, and thus $L=16.1$.

In this example, we select the gain matrix $\Gamma=\operatorname{diag}\left\{\begin{array}{ll}-1.1 & -0.9\end{array}\right\}$ with $\alpha=0.2$ and $\tau=0.1$ according to the corollary. Fig. 2 shows that the components of $\mathbf{e}$ approach zero.

(B) The Rössler system. The differential equations are

$$
\begin{aligned}
& \dot{x}=-y-z=: f_{1}(\mathbf{x}), \\
& \dot{y}=x+a y=: f_{2}(\mathbf{x}), \\
& \dot{z}=b+z(x-c)=: f_{3}(\mathbf{x}), \\
& \dot{\hat{x}}=-\hat{y}-\hat{z}, \\
& \dot{\hat{y}}=\hat{x}+a \hat{y} \\
& \dot{\hat{z}}=b+\hat{z}(\hat{x}-c), \\
& \left.\Delta \hat{x}\right|_{t=t_{i}}=\gamma_{1} e_{1}, \\
& \left.\Delta \hat{y}\right|_{t=t_{i}}=\gamma_{2} e_{2}, \\
& \left.\Delta \hat{z}\right|_{t=t_{i}}=\gamma_{3} e_{3}, \quad i=1,2, \ldots,
\end{aligned}
$$

where the parameters $a=b=0.2$ and $c=5.7$ with the initial condition $\left[\begin{array}{llllll}x_{0} & y_{0} & z_{0} & \hat{x}_{0} & \hat{y}_{0} & \hat{z}_{0}\end{array}\right]^{\mathrm{T}}=$ $\left[\begin{array}{llllll}1 & 1 & 1 & 0 & 0 & 0\end{array}\right]^{\mathrm{T}}$ ensure the existence of chaotic attractor. To apply the theorem, one needs to estimate a Lipschitz constant in advance. Applying the Cauchy-Schwarz inequality, for any $\mathbf{x}_{1}=\left[\begin{array}{lll}x_{1} & y_{1} & z_{1}\end{array}\right]^{\mathrm{T}}, \mathbf{x}_{2}=\left[\begin{array}{lll}x_{2} & y_{2} & z_{2}\end{array}\right]^{\mathrm{T}}$, we have 


\section{ARTICLE IN PRESS}
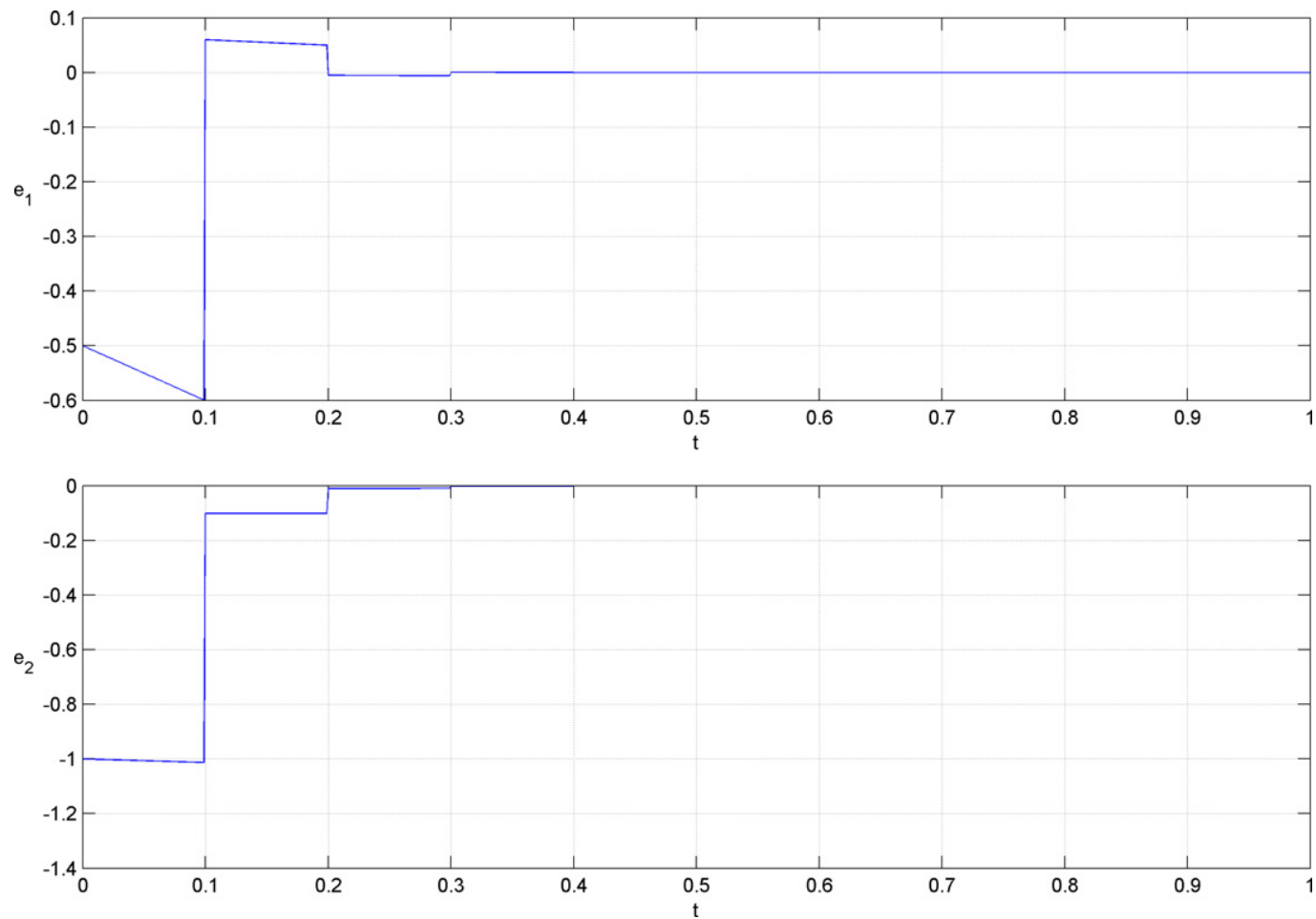

Fig. 2. The components of e for Duffing system approach zero as $\Gamma=\operatorname{diag}\{-1.1-0.9\}, \alpha=0.2$ and $\tau=0.1$.
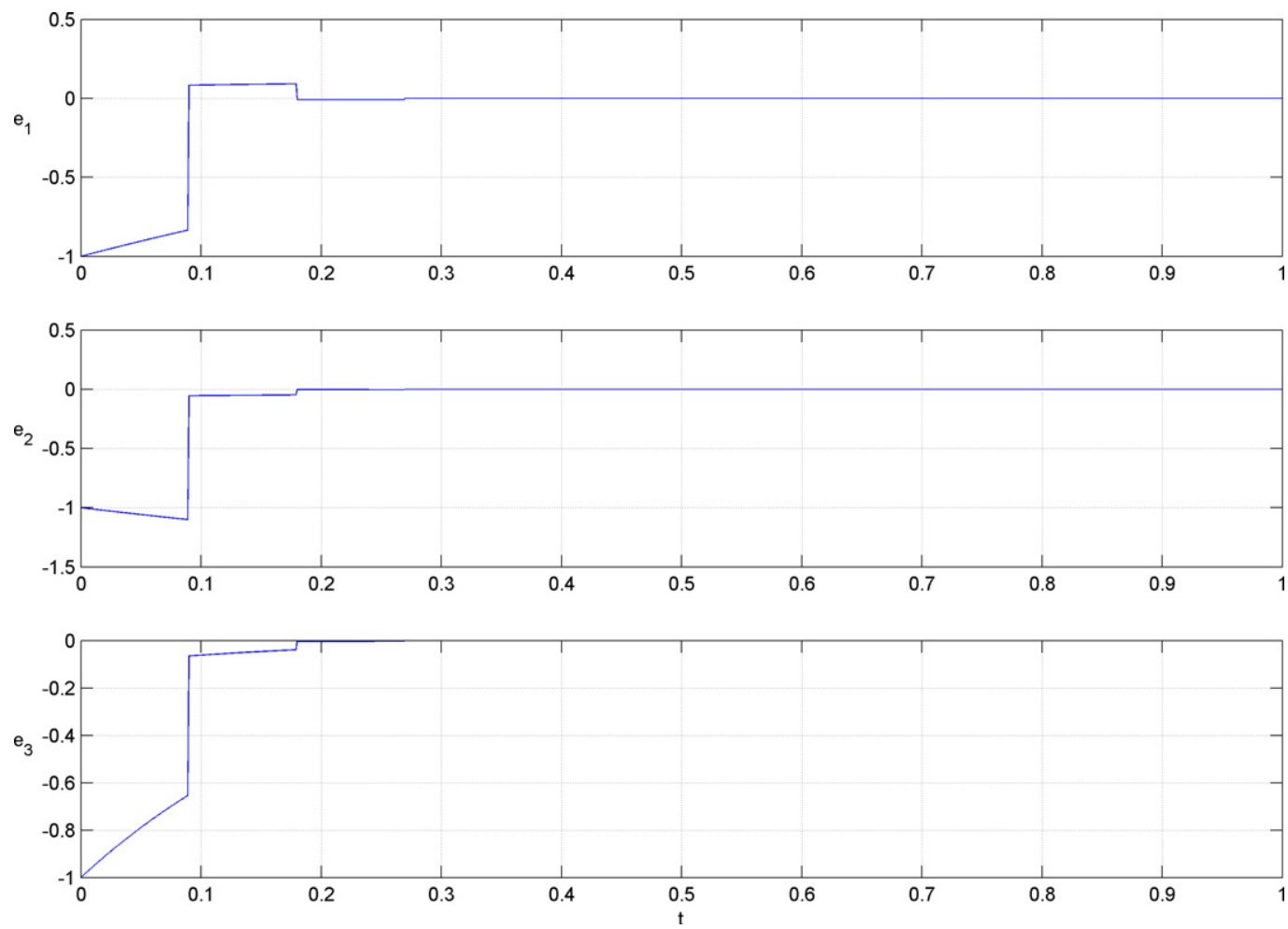

Fig. 3. Three components of $\mathbf{e}$ for Rössler system approach zero as $\Gamma=\operatorname{diag}\{-1.1-0.95-0.9\}, \alpha=0.2$ and $\tau=0.09$. 


$$
\begin{aligned}
\left|f_{1}\left(\mathbf{x}_{2}\right)-f_{1}\left(\mathbf{x}_{1}\right)\right| & \leqslant\left\|\left[\begin{array}{lll}
0 & -1 & -1
\end{array}\right]\right\|\left\|\mathbf{x}_{2}-\mathbf{x}_{1}\right\|, \\
\left|f_{2}\left(\mathbf{x}_{2}\right)-f_{2}\left(\mathbf{x}_{1}\right)\right| & \leqslant\left\|\left[\begin{array}{lll}
1 & a & 0
\end{array}\right]\right\|\left\|\mathbf{x}_{2}-\mathbf{x}_{1}\right\|, \\
\left|f_{3}\left(\mathbf{x}_{2}\right)-f_{3}\left(\mathbf{x}_{1}\right)\right| & =\left|z_{2} x_{2}-z_{1} x_{1}-c e_{3}\right| \\
& =\left|z_{2} x_{2}-z_{2} x_{1}+z_{2} x_{1}-z_{1} x_{1}-c e_{3}\right| \\
& =\left|z_{2} e_{1}+x_{1} e_{3}-c e_{3}\right| \\
& \leqslant\left\|\left[\begin{array}{lll}
B_{3} & 0 & B_{1}-c
\end{array}\right]\right\|\left\|\mathbf{x}_{2}-\mathbf{x}_{1}\right\|,
\end{aligned}
$$

where $\left|x_{i}(t)\right| \leqslant B_{1}, \quad\left|y_{i}(t)\right| \leqslant B_{2}, \quad\left|z_{i}(t)\right| \leqslant B_{3}, \forall t>t_{0}, i=1,2$. Hence, a Lipschitz constant can be elected to be $L=\sqrt{\left\|\left[\begin{array}{lll}0 & -1 & -1\end{array}\right]\right\|^{2}+\left\|\left[\begin{array}{lll}1 & a & 0\end{array}\right]\right\|^{2}+\left\|\left[\begin{array}{lll}B_{3} & 0 & B_{1}-c\end{array}\right]\right\|^{2}}$. From numerical simulation, we obtain $B_{1}=12$, $B_{2}=8, B_{3}=23$, and thus $L=23.55$.

In this example, we choose the gain matrix $\Gamma=\operatorname{diag}\left\{\begin{array}{lll}-1.1 & -0.95 & -0.9\end{array}\right\}$ with $\alpha=0.2$ and $\tau=0.09$ according to the corollary. Fig. 3 shows that three components of e approach zero.

\section{Concluding remarks}

The impulsive controller in the form of negative feedback has been shown to be an efficient method to achieve chaos synchronization. To ensure the exponential stability, we derived one single restriction (criterion) which describes a functional relationship among the Lipschitz constant $L$, the rate of synchronization $\alpha$, the impulse interval $t_{i}-t_{i-1}$, and the feedback gain matrix $\Gamma(i)$. The criterion is simple and easy to determine the admissible region of $t_{i}-t_{i-1}$ and $\gamma_{j}(i)$, if we are given an $L$ and decide an $\alpha$. The admissible region shrinks in size with increasing the Lipschitz constant $L$ or the rate of synchronization $\alpha$, whist the gain and interval margins are reduced correspondingly. The Duffing's two-well system and the Rössler system were simulated to demonstrate the theoretical analysis and effectiveness of the proposed impulsive controller.

\section{References}

[1] Fujisaka H, Yamada T. Preog. stability theory of synchronized motion in coupled-oscillator systems. Theor Phys 1983;69:32-47.

[2] Pecora LM, Carroll TL. Synchronization in chaotic systems. Phys Rev Lett 1990;64:821-4.

[3] Ott E, Grebogi C, Yorke JA. Controlling chaos. Phys Rev Lett 1990;64:1196-9.

[4] Vallejo JC, Mariño IP, Sanjuán MAF, Kurths J. Controlling chaos in a fluid flow past a movable cylinder. Chaos, Solitons \& Fractals 2003;15:255-63.

[5] Cuomo KM, Oppenheim AV. Circuit implementation of synchronized chaos with applications to communications. Phys Rev Lett 1993;71:65-8.

[6] Xie QX, Chen GR, Bollt EM. Hybrid chaos synchronization and its application in information processing. Math Comput Model 2002;35:145-63.

[7] Xiao JH, Hu G, Gao JH. Turbulence control and synchronization and controllable pattern formation. Int J Bifurcat Chaos 2000;10:655-60.

[8] Feudel F, Witt A, Gellert M, Kurths J, Grebogi C, Sanjuán MAF. Intersections of stable and unstable manifolds: the skeleton of Lagrangian chaos. Chaos, Solitons \& Fractals 2005;24:947-56.

[9] Pecora LM, Carroll TL. Synchronizing chaotic circuits. IEEE Trans Circuits Syst 1991;38:453-6.

[10] Rulkov NF, Sushchik MM, Tsimring LS. Generalized synchronization of chaos in directionally coupled chaotic systems. Phys Rev E 1995;51:980-94.

[11] Abarbanel HDI, Rulkov NF, Sushchik MM. Generalized synchronization of chaos: the auxiliary approach. Phys Rev E 1996;53:4528-35.

[12] Rosenblum MG, Pikovsky AS, Kurths J. Phase synchronization of chaotic oscillators. Phys Rev Lett 1996;76:1804-7.

[13] Rosenblum MG, Pikovsky AS, Kurths J. From phase to lag synchronization in coupled chaotic oscillators. Phys Rev Lett 1997;78:4193-6.

[14] Voss HU. Anticipating chaotic synchronization. Phys Rev E 2000;61:5115-9.

[15] Shahverdiev EM, Nuriev RA, Hashimov RH, Shore KA. Parameter mismatched, variable delay times and synchronization in time delayed systems. Chaos, Solitons \& Fractals 2005;25:325-31.

[16] Voss H, Kurths J. Reconstruction of nonlinear time-delayed feedback models from optical data. Chaos, Solitons \& Fractals 1999;10:805-9.

[17] Yang T, Chua LO. Impulsive stabilization for control and synchronization of chaotic systems: theory and application to secure communication. IEEE Trans Circuits Syst-I 1997;44:976-88. 
[18] Li CD, Liao XF. Complete and lag synchronization of hyperchaotic systems using small impulses. Chaos, Solitons \& Fractals 2004;22:857-67.

[19] Sun JT, Zhang YP, Qiao F, Wu QD. Some impulsive synchronization criterions for coupled chaotic systems via unidirectional linear error feedback approach. Chaos, Solitons \& Fractals 2004;19:1049-55. 